\title{
Etude de la valeur agronomique en plante entière au stade de récolte ensilage de différentes varié- tés de maïs grain sélectionnées entre 1950 et 1980
}

\author{
Y. BARRIÈRE, A. GALLAIS $\left({ }^{*}\right)$, M. DERIEUX $\left({ }^{* *}\right)$, A. PANOUILLÉ $(* * *)$ \\ I.N.R.A., Station d'Amélioration des Plantes Fourragères, Centre de Recherches de Poitou-Charentes, \\ $F 86600$ Lusignan. \\ (*) C.E.B.A., G.I.S.-Moulon, F 91190 Gif-sur-Yvette \\ (**) I.N.R.A., Amélioration des Plantes, F 80200 Péronne \\ (***) I.N.R.A., Station Expérimentale du Maïs, F 40590 St-Martin-de-Hinx
}

Mots clés additionnels : Biomasse, maï, maïs ensilage, digestibilité in vitro, hybride, lignée, progrès génétique.

Eighteen early and mid-early maize hybrids grown in France since 1950 have been compared in order to study the correlative variation in whole plant value at the time of the harvest for silage, after 30 years of breeding for grain yield ( 2 years, 3 densities, 1 to 3 locations). The results are relevant mainly for the chosen hybrids and locations, but general trends also emerge. For the early hybrids, biomass improvement was $0.07 \mathrm{t} / \mathrm{ha} / \mathrm{year}$, which resembles the grain improvement measured $(0.08 \mathrm{t} / \mathrm{ha} /$ year $)$, even if the locations were not exactly the same for the grain and silage trials. For mid-early hybrids, studied for the production of silage in only one location quite favourable for maize crops, biomass evolution was negative $(-0.02 \mathrm{t} / \mathrm{ha} /$ year $)$, whereas grain improvement observed elsewhere was positive $(0.04 \mathrm{t} / \mathrm{ha} /$ year $)$. In the first case, biomass improvement was complelely used for grain improvement ; in the second, grain improvement was related to an increase in harvest index, as shown for example in the case of wheat. Observed differences in feeding values, evaluated by in vitro methods, were not clearly related to the year of hybrid release ; nor were differences in individual values of inbreds for whole plant yield. This study confirms that it would be more efficient to breed maize for silage by use of specific materials and tests, rather than use of grain hybrids in forage tests.

Additional key words : Biomass, corn, silage maize, in vitro digestibility, hybrid, inbred line, genetic improvement. 


\section{INTRODUCTION}

L'extension du maïs en dehors du Sud-Ouest de la France est récente puisqu'elle a débuté à partir des années 1950 avec l'introduction des hybrides du type Wisconsin $240,255,355$ et 416 . Cette culture s'est ensuite étendue à des zones plus fraîches avec la création des lignées cornées (comme F7, F2 et Ep1) et la diffusion d'hybrides cornés $\times$ dentés. Des essais multilocaux ont été réalisés à l'initiative de l'I.N.R.A. pour apprécier le progrès génétique réalisé depuis cette époque, qui montrent que l'augmentation du rendement en grain se situe autour de 0,6 quintal par hectare et par an sur les 30 dernières années (période 1950-1980) (DERIEUX et al., 1987). Parallèlement, des essais moins nombreux ont été réalisés pour mesurer l'évolution de la productivité en biomasse aérienne sur les mêmes génotypes, en se limitant aux deux groupes précoces et demi-précoces. Outre son intérêt théorique, cette étude a aussi des implications pratiques puisque ce sont actuellement des variétés dont les géniteurs ont subi un crible grain pendant une trentaine d'années qui sont utilisées en ensilage, activité qui représente pratiquement la moitié des surfaces en maiis en France comme dans la CEE.

\section{MATÉRIEL ET MÉTHODES}

Les expérimentations ont été réalisées en 1983 et 84 sur les domaines I.N.R.A. de Mons (Somme, Nord de la France), du Gis-Moulon (Essonne, Bassin parisien) et de Lusignan (Vienne, Centre-Ouest de la France). 18 hybrides inscrits entre 1951 et 1981 ont été répartis en deux groupes de précocité, les hybrides LG11, Liza et Dea en 1984 étant répétés dans les deux groupes. Le groupe précoce comprenait ainsi les hybrides Wisconsin 240, Wisconsin 255 (inscrits en 1950), INRA 200 (1961), INRA 258 (1962), LG11 (1970), Cargill Primeur 170 (1973), Liza (1978), Brulouis INRA 180 (1980), Leader Pau 207 (1980), HTV 270, hybride inscrit en 1985 sous le nom de Browning INRA 150, variété plus précoce que l'ensemble de ce groupe, et Dea (1980), variété de référence du groupe demiprécoce. Le groupe demi-précoce comprenait Wisconsin 355, Wisconsin 416 (inscrits en 1950), INRA 260 (1960), INRA 420 (1962), INRA 430 (1961), LG11 (1970), Star 304 (1969), Liza (1978), Bruex (1980) et Dea (1980). Les essais des hybrides précoces ont été réalisés sur les 3 lieux, alors que les essais du groupe des demi-précoces ne l'ont été qu'à Lusignan.

Les essais ont été semés suivant un protocole en bloc à 3 répétitions d'une ou deux lignes de 4 ou $5 \mathrm{~m}$ à trois densités. Les productivités et précocités (teneur en matière sèche) en plante entière au stade de récolte ensilage ont été mesurées sur tous les lieux avec de plus des notations de peuplement et de floraison au Moulon et à Lusignan, ainsi que certaines observations complémentaires sur ces deux lieux (hauteur totale des plantes...).

Les façons culturales sont celles de la pratique courante d'exploitations modernes (insecticide localisé dans la raie de semis, désherbage), avec des doses d'azote comprises entre 160 et 180 unités par hectare selon les lieux et les années. Les précédents culturaux étaient un blé à Mons en 1983 et 84 ainsi qu'au Moulon en 1983, un maïs au Moulon en 1984 et à Lusignan en 1985, une prairie de fétuque élevée à Lusignan en 1983 et une orge à Lusignan en 1984. Pour compenser un déficit hydrique d'été important à Lusignan, il a été apporté sur ce lieu une irrigation de $60 \mathrm{~mm}$ d'eau chaque année.

Les semences des variétés qui ne sont plus commercialisées ont été produites par la Station expérimentale du maïs de l'I.N.R.A., à St-Martin-de-Hinx (Landes).

Les analyses de variance ont été réalisées avec le programme Anvarm de la bibliothèque Amance (BACHACOU et al., 1981) avec :

- un facteur lieu,

- un facteur année,

- un facteur densité,

- un facteur bloc hiérarchisé au facteur densité,

- un facteur génotype,

- une interaction lieu $\times$ densité,

- une interaction lieu $x$ génotype,

- une interaction année $\times$ génotype,

- une interaction densité $\times$ génotype.

Pour les essais des génotypes demi-précoces à Lusignan, les valeurs du rendement ont été ajustées des covariances résiduelles rendement $x$ peuplement, en raison de peuplements parfois irréguliers ( $\mathrm{r}$ intra rendement $\times$ peuplement ramenée de 0,35 à 0,0$)$. Pour les essais des génotypes précoces, cet ajustement n'a pas été réalisé en raison de la faible corrélation intra rendement $\times$ peuplement sur le Moulon et Lusignan $(\mathrm{r}=-0,18)$ et de l'absence de comptages de plantes à floraison à Mons.

Pour confirmer les résultats déjà obtenus, et conforter l'aspect valeur alimentaire in vitro des fourrages récoltés ébauché sur les génotypes précoces en 1984 à Lusignan, des essais supplémentaires y ont été réalisés en 1985 sur certains hybrides demi-précoces dans les mêmes conditions que précédemment, à la densité la plus élevée. De plus, pour étudier l'évolution de la valeur propre des lignées au cours des cycles d'amélioration, des essais de valeur plante entière d'un certain nombre des lignées constitutives ont également été réalisés en 1985 à Lusignan, suivant un protocole en bloc à 3 répétitions d'une ligne de $5 \mathrm{~m}$. La densité effective à la récolte était de 57500 plantes par hectare, et pour éviter les effets de bordure, chaque lignée était séparée de la suivante par une ligne de la lignée précoce F272.

\section{RÉSULTATS ET DISCUSSION}

Les résultats des différents essais sont donnés dans les tableaux 1 et 2 pour les rendements et précocités en plante entière. Une synthèse par génération d'hybride est présentée dans le tableau 3.

Les différents effets principaux (lieu, année, densité, génotype) sont significatifs, très généralement au seuil de 1 p. 100 , quelquefois au seuil de 5 p. 100 pour les 2 caractères considérés. L'interaction lieu $\times$ génotype est significative (au seuil de 1 p. 100) pour le rendement dans les essais de génotypes précoces, alors que généralement toutes les interactions envisagées sont faibles et que, en particulier, aucune des interactions densité $\times$ génotype n'est significative. 
TABLEAU 1

Hybrides précoces. Analyse de variance et moyennes marginales pour le rendement et la précocité en plante entière.

Early hybrids. Analysis of variance for yield and dry-matter content of the whole plant.

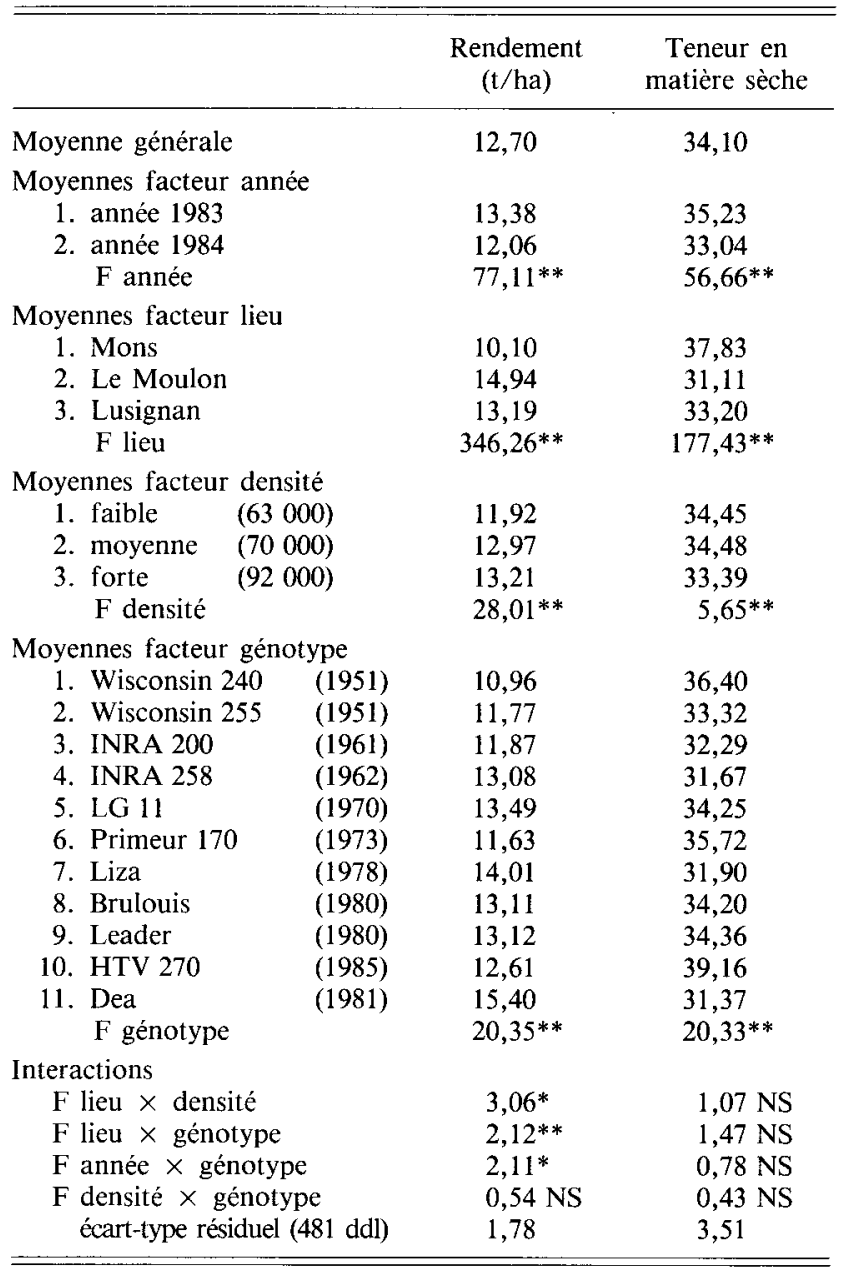

** significatif au seuil de $1 \%$.

* significatif au seuil de $5 \%$.

Le «progrès génétique » en productivité plante entière réalisé entre 1950 et 1980 , et tel qu'il est estimé à partir des génotypes précoces retenus, est en moyenne sur les 3 lieux de 2,07 tonnes de matière sèche par hectare, la précocité des hybrides étant comparable. Ce gain sur 30 ans se répartit en fait pour moitié entre 1950 et 1960 d'une part (environ 1 tonne) et 1970 et 1980 d'autre part (environ 1 tonne également). Par ailleurs, l'estimation de ce gain est variable sur les différents lieux. Le progrès mesuré est nettement plus important dans les milieux frais (Mons, Le Moulon) où il atteint 2,5 tonnes en 30 ans, alors qu'il paraît plus faible dans un milieu non limitant en température d'été et d'arrière-saison pour ces génotypes (Lusignan) où il n'atteint que 1,15 tonnes. Plus de la moitié du progrès observé serait donc lié à des adaptations aux faibles températures, et non pas à des augmentations de production de biomasse. Par ailleurs, les premiers génotypes adaptés aux conditions françaises (hybrides de 1960) ne diffèrent pas en productivité en plante entière des hybrides modernes (hybride de 1980). Une part notable du gain de valeur agronomi-
TABLEAU 2

Hybrides demi-précoces. Analyse de variance et moyennes marginales pour le rendement et la précocité en plante entière.

Mid-early hybrids. Analysis of variance for yield and dry-matter content of the whole plant.

\begin{tabular}{|c|c|c|}
\hline & $\begin{array}{l}\text { Rendement } \\
\text { (t/ha) }\end{array}$ & $\begin{array}{l}\text { Teneur en } \\
\text { matière sèche }\end{array}$ \\
\hline Moyenne générale & 13,99 & 29,23 \\
\hline $\begin{array}{l}\text { Moyennes facteur année } \\
\text { 1. année } 1983 \\
\text { 2. année } 1984 \\
\text { F année }\end{array}$ & $\begin{array}{l}12,97 \\
14,91 \\
79,84^{* *}\end{array}$ & $\begin{array}{l}29,57 \\
28,80 \\
5,62 *\end{array}$ \\
\hline 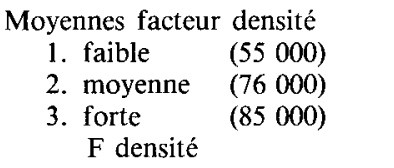 & $\begin{array}{l}12,69 \\
14,25 \\
14,87 \\
36,10^{* *}\end{array}$ & $\begin{array}{r}29,83 \\
29,31 \\
28,56 \\
6,72 * *\end{array}$ \\
\hline 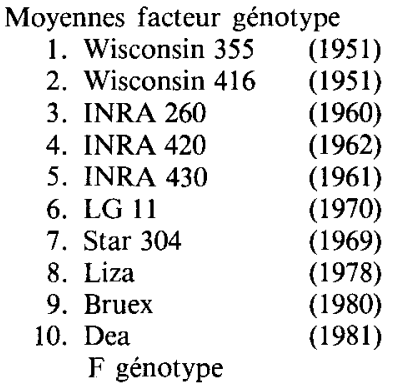 & $\begin{array}{c}13,20 \\
15,18 \\
12,69 \\
13,92 \\
15,58 \\
12,81 \\
15,32 \\
13,14 \\
13,60 \\
13,83 \\
9,76^{* *}\end{array}$ & $\begin{array}{l}29,47 \\
29,99 \\
28,56 \\
26,04 \\
28,73 \\
32,49 \\
27,97 \\
30,38 \\
28,80 \\
29,99 \\
14,06^{* *}\end{array}$ \\
\hline $\begin{array}{l}\text { Interactions } \\
\qquad \mathrm{F} \text { année } \times \text { génotype } \\
\mathrm{F} \text { densité } \times \text { génotype } \\
\text { écart-type résiduel }(133 \mathrm{ddl})\end{array}$ & $\begin{array}{l}1,12 \mathrm{NS} \\
0,64 \mathrm{NS} \\
1,45\end{array}$ & $\begin{array}{l}2,14^{*} \\
1,14 \mathrm{NS} \\
1,91\end{array}$ \\
\hline
\end{tabular}

** significatif au seuil de $1 \%$.

* significatif au seuil de $5 \%$.

que ainsi observé dans les milieux frais est sans doute due au remplacement des hybrides dentés purs comme Wisconsin 240 et Wisconsin 255 par des hybrides cornés $\times$ dentés plus tolérants aux basses températures comme INRA 200 et surtout INRA 258.

L'hybride HTV 270 (1985, Browning INRA 150) qui a une productivité en plante entière en moyenne sur les 3 lieux égale à celle des hybrides de la génération 1960, et 1 demi-tonne environ inférieure à celle des hybrides de la génération 1980, présente en revanche une amélioration très importante de la précocité, en étant en particulier 7 points plus précoce que les hybrides du type INRA 200 et INRA 258. Ceci représente une amélioration de la précocité un peu inférieure à un tiers de point par an pendant 25 ans, tout en maintenant la productivité en biomasse. L'hybride Dea, qui est en principe plus tardif, a une productivité en plante entière supérieure aux hybrides de ce groupe.

Pour les hybrides demi-précoces étudiés seulement à Lusignan, il n'est pas mis en évidence de « progrès génétique » entre 1950 et 1980 avec ces génotypes, voire au contraire un léger déclin en productivité, à précocité en plante entière égale. L'hybride Wisconsin 416 apparaît même meilleur en production de biomasse que de très bons hybrides grains actuels.

Contrairement à ce qui est classiquement admis et observé dans les essais de maïs grain, il n'apparaît pas dans ces essais de progrès en adaptation aux fortes 
TABLEAU 3

Approche de la variation observée pour la productivité et la précocité en plante entière pour des génotypes précoces et demi-précoces inscrits entre 1950 et 1980.

Observed genetic variation for whole-plant yield between early or mid-early maize hybrids released from 1950 to 1980.

\begin{tabular}{|c|c|c|c|c|c|}
\hline & & & $\begin{array}{l}\text { Rendement } \\
\text { t/ha }\end{array}$ & $\begin{array}{l}\text { Evolution du } \\
\text { rendement à } \\
\text { partir de } 1950\end{array}$ & $\% \mathrm{M}$. sèche \\
\hline \multicolumn{6}{|c|}{ Hybrides précoces } \\
\hline 1950 & $\begin{array}{l}\text { Wisconsin } 240 \\
\text { Wisconsin } 255\end{array}$ & $\begin{array}{c}\text { Moyenne des } 3 \text { lieux } \\
\text { - Mons } \\
\text { - Le Moulon } \\
\text { - Lusignan }\end{array}$ & $\begin{array}{r}11,37 \\
8,69 \\
13,14 \\
12,39\end{array}$ & $\begin{array}{l}- \\
- \\
-\end{array}$ & $\begin{array}{l}34,86 \\
37,93 \\
32,56 \\
33,93\end{array}$ \\
\hline 1960 & $\begin{array}{l}\text { INRA } 200 \\
\text { INRA } 258\end{array}$ & $\begin{array}{c}\text { Moyenne des } 3 \text { lieux } \\
\text { - Mons } \\
\text { - Le Moulon } \\
\text { - Lusignan }\end{array}$ & $\begin{array}{r}12,48 \\
9,82 \\
14,34 \\
13,37\end{array}$ & $\begin{array}{l}1,11 \\
1,13 \\
1,20 \\
0,98\end{array}$ & $\begin{array}{l}31,98 \\
35,40 \\
28,91 \\
31,45\end{array}$ \\
\hline 1970 & $\begin{array}{l}\text { LG } 11 \\
\text { Primeur }\end{array}$ & $\begin{array}{c}\text { Moyenne des } 3 \text { lieux } \\
\text { - Mons } \\
\text { - Le Moulon } \\
\text { - Lusignan }\end{array}$ & $\begin{array}{r}12,56 \\
9,76 \\
15,36 \\
12,70\end{array}$ & $\begin{array}{l}1,19 \\
1,07 \\
2,22 \\
0,31\end{array}$ & $\begin{array}{l}34,99 \\
38,70 \\
31,90 \\
34,18\end{array}$ \\
\hline 1980 & $\begin{array}{l}\text { Liza } \\
\text { Brulouis } \\
\text { Leader }\end{array}$ & $\begin{array}{c}\text { Moyenne des } 3 \text { lieux } \\
\text { - Mons } \\
\text { - Le Moulon } \\
\text { - Lusignan }\end{array}$ & $\begin{array}{l}13,44 \\
11,06 \\
15,77 \\
13,54\end{array}$ & $\begin{array}{l}2,07 \\
2,37 \\
2,63 \\
1,15\end{array}$ & $\begin{array}{l}33,49 \\
37,67 \\
29,96 \\
32,68\end{array}$ \\
\hline \multicolumn{6}{|c|}{ Hybrides demi-précoces } \\
\hline 1950 & $\begin{array}{l}\text { Wisconsin } 355 \\
\text { Wisconsin } 416\end{array}$ & & 14,19 & - & 29,73 \\
\hline 1960 & $\begin{array}{l}\text { INRA } 260 \\
\text { INRA } 420 \\
\text { INRA } 430\end{array}$ & & 14,08 & $-0,08$ & 27,78 \\
\hline 1970 & $\begin{array}{l}\text { LG } 11 \\
\text { Star } 304\end{array}$ & & 14,07 & $-0,09$ & 30,23 \\
\hline 1980 & $\begin{array}{l}\text { Liza } \\
\text { Bruex } \\
\text { Dea }\end{array}$ & & 13,52 & $-0,67$ & 29,72 \\
\hline
\end{tabular}

densités pour la productivité en plante entière puisque les interactions génotypes $x$ densité ne sont pas significatives.

Les observations complémentaires réalisées sur certains essais montrent une amélioration de la vigueur au départ et de la résistance à la verse ou à la casse pour les génotypes précoces et demi-précoces. En revanche, certains caractères morphologiques étudiés comme la hauteur d'insertion de l'épi, la surface de la feuille de l'épi et la prolificité en épis ne semblent pas avoir varié au cours des générations de sélection (Le Moulon, 1983-84, hybrides précoces). Toutefois une plus grande hauteur des génotypes précoces modernes a été observée au Moulon en 1984, avec au contraire une plus faible hauteur des demi-précoces modernes à Lusignan en 1983.

Les observations supplémentaires réalisées à Lusignan en 1985 sur 7 des hybrides demi-précoces (tabl. 4) confirment les résultats des deux années précédentes, à savoir l'absence d'évolution en biomasse dans ce milieu, à précocité égale, et une amélioration de la vigueur au départ apparemment un peu plus faible cette année-là que précédemment.

L'approche des aspects qualité (tabl. 5) montre qu'il ne semble pas y avoir eu d'évolution de la teneur globale en fibres $(\mathrm{ADF}=$ acid detergent fiber, mesure

\section{TABLEAU 4}

Hybrides demi-précoces. Observations supplémentaires réalisées à Lusignan en 1985. Rendement, précocité et vigueur au départ de 7 génotypes.

Mid-early hybrids. Yield, earliness, and early vigor observed for 7 genotypes in Lusignan (1985).

\begin{tabular}{llcc}
\hline & Rendement $\%$ M. sèche & $\begin{array}{c}\text { Vigueur } \\
\text { au depart }\end{array}$ \\
\hline 1. Wisconsin 355 & 13,82 & 34,28 & 2,3 \\
2. Wisconsin 416 & 15,88 & 37,10 & 3,0 \\
3. INRA 260 & 14,15 & 35,87 & 3,7 \\
5. INRA 430 & 15,68 & 31,05 & 3,2 \\
7. Star 304 & 15,84 & 36,08 & 3,0 \\
9. Bruex & 13,81 & 36,35 & 3,5 \\
10. Dea & 15,46 & 36,55 & 3,2 \\
F génotypes & & & \\
(6 répétitions) & $2,21 \mathrm{NS}$ & $2,00 \mathrm{NS}$ & $3,38^{*}$ \\
\hline
\end{tabular}

* significatif au seuil de $5 \%$.

globale des lignines et celluloses) entre génotypes anciens et modernes, et cela malgré un progrès pour la résistance à la verse à la récolte (DERIEUX et al., 1987). Toutefois, il y a une tendance à la dimi- 
TABLEAU 5

Valeur alimentaire in vitro mesurée sur les hybrides cultivés à forte densité (1984-85, Lusignan).

Feeding value characteristics measured in vitro with hybrids of the highest density (1984-85, Lusignan).

\begin{tabular}{|c|c|c|c|c|c|}
\hline & \multirow[b]{2}{*}{$\mathrm{ADF}$} & \multirow[b]{2}{*}{ Celluloses } & \multirow[b]{2}{*}{ Glucides } & \multicolumn{2}{|c|}{ Digestibilité } \\
\hline & & & & Jus de rumen & APC \\
\hline \multicolumn{6}{|c|}{ Essai 1984 - Lusignan } \\
\hline 1. Wisconsin 240 & 24,67 & 20,27 & - & 58,60 & 75,93 \\
\hline 2. Wisconsin 255 & 20,57 & 16,97 & - & 63,93 & 77,90 \\
\hline 3. INRA 200 & 19,33 & 16,27 & - & 67,33 & 85,00 \\
\hline 4. INRA 258 & 19,20 & 15,93 & - & 66,60 & 81,90 \\
\hline 5. LG 11 & 17,83 & 15,27 & - & 68,77 & 85,83 \\
\hline 6. Primeur & 21,23 & 18,20 & - & 62,93 & 82,87 \\
\hline 7. Liza & 20,63 & 17,37 & - & 65,37 & 80,13 \\
\hline 8. Brulouis & 20,53 & 17,40 & - & 65,90 & 84,17 \\
\hline 9. Leader & 19,57 & 16,57 & - & 65,10 & 85,10 \\
\hline 10. HTV 270 & 20,30 & 17,47 & - & 63,67 & 80,83 \\
\hline 11. Dea & 18,43 & 15,70 & - & 69,60 & 83,20 \\
\hline F génotypes & $1,87 \mathrm{NS}$ & $1,87 \mathrm{NS}$ & - & $2,39^{*}$ & $2,54^{*}$ \\
\hline \multicolumn{6}{|c|}{ Essai 1985 - Lusignan } \\
\hline 1. Wisconsin 355 & 24,87 & 21,97 & 5,90 & 62,00 & 79,00 \\
\hline 2. Wisconsin 416 & 26,83 & 24,67 & 5,47 & 58,86 & 79,30 \\
\hline 3. INRA 260 & 22,37 & 20,37 & 5,73 & 65,37 & 84,07 \\
\hline 5. INRA 430 & 27,83 & 23,90 & 6,93 & 59,53 & 78,23 \\
\hline 7. Star 304 & 24,53 & 22,27 & 5,47 & 62,90 & 84,30 \\
\hline 9. Bruex & 23,37 & 18,97 & 4,57 & 63,60 & 83,77 \\
\hline 10. Dea & 25,00 & 20,67 & 4,97 & 61,90 & 81,53 \\
\hline F génotypes & $1,54 \mathrm{NS}$ & $8,37^{* *}$ & $0,77 \mathrm{NS}$ & $2,27 \mathrm{NS}$ & $1,95 \mathrm{NS}$ \\
\hline
\end{tabular}

** significatif au seuil de $1 \%$.

* significatif au seuil de $5 \%$.

nution de la teneur en cellulose significative chez les génotypes demi-précoces ; ce qui voudrait dire que les génotypes modernes sont plus riches en lignines et plus pauvres en celluloses, observations qui vont dans le même sens que l'augmentation du rendement en grain, à production de biomasse équivalente ou supérieure, avec une amélioration de la résistance mécanique des tiges. Les différences de digestibilité in vitro observées (méthode jus de rumen, TILLEY \& TERRY, 1963, ou méthode enzymatique APC, LILA et al., 1986) ne semblent pas liées, quand elles sont significatives, à la génération des hybrides, si ce n'est une faible valeur pour Wisconsin 240 .

L'étude des lignées en valeur propre (tabl. 6) montre que le rendement en biomasse de celle-ci, dans le milieu Lusignan, est pratiquement indépendant de leur génération. Des lignées anciennes demi-précoces comme A374, ia 153 ont une productivité plante entière non différente de celle de lignées modernes comme MBS 847 , à précocité égale, alors que parmi les lignées précoces F252 paraît un peu plus productive que les lignées anciennes comparables. L'amélioration principale de la valeur propre des lignées aurait donc surtout porté sur la résistance à la verse en végétation et la résistance aux pourritures de tiges, éventuellement sur la productivité en grain.

\section{CONCLUSIONS}

Avant de comparer les évolutions mesurées en productivité en grain et en plante entière, il faut noter que ces deux mesures n'ont pas été réalisées au même stade. Toutefois, le stade de récolte ensilage correspond pratiquement au palier de productivité en matière sèche des génotypes, à partir duquel les augmentations ultérieures de rendement en grain sont dues à des transferts d'assimilats à partir des tiges et des racines. On peut donc penser que la sousestimation éventuelle du rendement total en biomasse devrait être faible ou très faible, et cela d'autant plus que les teneurs en matière sèche des plantes ensilées sont proches de 30 p. 100, ou supérieures, en particulier pour les hybrides précoces. En revanche, cette remarque ne change en rien les conclusions sur l'évolution corrélative observée pour la valeur en plante entière de génotypes triés sur leur valeur en grain. Un second biais dans cette comparaison peut être dû à la non-similarité des réseaux d'essais, plus complets pour les essais grains, en particulier pour les hybrides demiprécoces observés en ensilage seulement à Lusignan, milieu dont l'été et l'arrière-saison sont généralement favorables au maîs.

Les essais du réseau grain montrent que les progrès génétiques réalisés pour la productivité en grain entre 1950 et 1980 se situent respectivement autour de 0,08 et $0,04 \mathrm{t}$ de grain par hectare et par an pour les génotypes précoces et demi-précoces, progrès qui représentent la moitié environ des gains agronomiques moyens observés en culture pendant la même période (DERIEUX et al., 1987). Les "progrès » corrélatifs en biomasse qui s'observent chez les génotypes précoces au stade ensilage sont du même ordre qu'en grain, $0,07 \mathrm{t} / \mathrm{ha} / \mathrm{an}$ en moyenne, $0,08 \mathrm{t} / \mathrm{ha} / \mathrm{an}$ dans les deux milieux les plus difficiles et $0,04 \mathrm{t} / \mathrm{ha} / \mathrm{an}$ dans le milieu le plus favorable; ce qui signifierait que pour 
TABLEAU 6

Etude en valeur propre de lignées constitutives des hybrides précoces et demi-précoces. Individual value of some inbreds used in early and mid-early hybrids.

\begin{tabular}{|c|c|c|c|c|c|c|}
\hline & $\begin{array}{l}\text { Année de } \\
\text { fixation }\end{array}$ & $\begin{array}{l}\text { Générations des } \\
\text { hybrides étudiés } \\
\text { utilisant la lignée }\end{array}$ & $\begin{array}{l}\text { Rendement plante } \\
\text { entière }(\mathrm{t} / \mathrm{ha})\end{array}$ & $\begin{array}{l}\text { Rendement corrigé } \\
\text { de la date de } \\
\text { floraison }(\mathrm{t} / \mathrm{ha})\end{array}$ & $\%$ M. sèche & $\begin{array}{c}\text { Date floraison } \\
\text { femelle } \\
\text { (quantième) }\end{array}$ \\
\hline WD & $1940 \pm 5$ & 1950 & 6,06 & 6,44 & 33,84 & 213 \\
\hline W9 & $1940 \pm 5$ & 1950 & 7,47 & 7,60 & 36,84 & 217 \\
\hline WH & $1940 \pm 5$ & $1950-1960$ & 6,33 & 6,80 & 29,01 & 212 \\
\hline WJ & $1040 \pm 5$ & $1950-1960$ & 4,78 & 4,69 & 29,58 & 220 \\
\hline $\mathrm{F} 2$ & 1956 & $1960-1970-1980$ & 5,43 & 6,14 & 31,46 & 209 \\
\hline F7 & 1957 & $1960-1970$ & 4,26 & 5,02 & 39,30 & 208 \\
\hline Epl & $1950 \pm 5$ & 1960 & 7,92 & 8,55 & 28,01 & 210 \\
\hline F115 & 1955 & 1960 & 7,20 & 7,45 & 28,94 & 215 \\
\hline W33 & & 1960 & 5,20 & 5,38 & 29,84 & 216 \\
\hline W401 & & 1970 & 5,41 & 5,29 & 31,70 & 220 \\
\hline F192 & 1968 & 1980 & 5,05 & 5,78 & 34,31 & 209 \\
\hline $\mathrm{F} 252$ & 1976 & 1980 & 8,18 & 8,81 & 35,81 & 210 \\
\hline WM13 & & 1950 & 8,20 & 7,82 & 25,69 & 223 \\
\hline W25 & & 1950 & 6,89 & 5,98 & 22,83 & 230 \\
\hline ia 153 & & 1950 & 7,67 & 7,81 & 27,06 & 216 \\
\hline A 374 & & $1950-1960$ & 7,91 & 7,81 & 27,66 & 220 \\
\hline W 182E & & 1960 & 8,88 & 8,50 & 23,29 & 223 \\
\hline W153R & & 1960 & 4,25 & 3,79 & 23,81 & 225 \\
\hline F52 & 1958 & 1960 & 3,80 & 3,72 & 26,59 & 219 \\
\hline F432 & 1960 & 1960 & 5,70 & 5,12 & 26,22 & 226 \\
\hline A 632 & $1950 \pm 5$ & 1970 & 9,27 & 8,26 & 26,43 & 232 \\
\hline A635 & $1950 \pm 5$ & 1970 & 5,72 & 4,63 & 27,93 & 233 \\
\hline W117 & & 1970 & 6,97 & 7,05 & 27,26 & 217 \\
\hline$F 230$ & 1972 & 1980 & 6,25 & 5,82 & 22,33 & 224 \\
\hline MBS 847 & & 1980 & 9,12 & 8,36 & 27,03 & 228 \\
\hline $\mathbf{F}$ génotypes & & & $6,21^{* *}$ & $5,34^{* *}$ & $8,57 * *$ & $20,31 * *$ \\
\hline
\end{tabular}

** significatif au seuil de $1 \%$.

ce groupe d'hybrides les progrès en biomasse ont été complètement réinvestis en grain. Pour les hybrides demi-précoces, les observations conduisent à une réduction de biomasse de $0,02 \mathrm{t} / \mathrm{ha} / \mathrm{an}$, mesurée au stade ensilage à 29 p. 100 de matière sèche dans les fourrages récoltés. Les progrès en rendement grain ne seraient alors dus qu'à des augmentations des indices de récolte, même si la valeur obtenue peut être considérée comme minimale. Si l'on ne prend en compte que la meilleure densité (qui est toujours la plus élevée), les progrès mesurés en biomasse sont les mêmes, $0,07 \mathrm{t} / \mathrm{ha} / \mathrm{an}$ et $-0,03 \mathrm{t} / \mathrm{ha} / \mathrm{an}$ pour les génotypes précoces et demi-précoces respectivement. Cette différence d'évolution entre les génotypes précoces et demi-précoces, avec un progrès en productivité en grain lié à une augmentation de la biomasse et de l'indice de récolte, ou de l'indice de récolte seulement s'explique peut-être par des niveaux différents des indices de récolte au départ. Dans un premier temps, et avec des indices plutôt faibles, il est possible de gagner en rendement en grain en modifiant seulement ces indices; ensuite il est obligatoire d'augmenter aussi la production de biomasse, surtout s'il y a un tri simultané pour la qualité des tiges à la récolte. La résistance à la verse à maturité et/ou à la verse due aux pourritures des tiges a d'ailleurs été beaucoup améliorée (DERIEUX et al., 1987), mais les essais récoltés au stade ensilage ne permettent pas de la mettre en évidence, et ceci explique sans doute, en partie au moins, l'absence d'interaction densité $\times$ génotype pour la productivité.
L'absence d'évolution des digestibilités des plantes entières, malgré une augmentation très probable des teneurs en grain au stade de récolte ensilage, confirme l'importance des interrelations amidon $\times$ celluloses ou plus généralement amidon $\times$ fibres dans l'établissement de la résultante digestibilité. D'autre part, l'absence d'évolution de productivité en biomasse des lignées constitutives de ces hybrides n'est pas contradictoire avec les méthodes de sélection généalogique employées, qui privilégient en valeur propre le phénotype de l'épi et la résistance à la verse du matériel en cours de sélection.

Ces conclusions sont sans doute liées à la fois aux génotypes retenus et aux réseaux d'essais choisis, mais, d'une manière générale, elles vont dans le même sens que des travaux similaires. Sur maïs, les études concernant le progrès génétique ne semblent avoir pris en compte que l'aspect grain, et elles mettent en évidence un progrès voisin de $0,1 \mathrm{t} / \mathrm{ha} / \mathrm{an}$ sur 50 ans dont 33 à 89 p. 100 est attribué à des effets génétiques (CASTLEBERRY et al., 1984 ; DUVICK, 1984 ; RUSSEL, 1984). Sur blé, AUSTIN et al. (1980) montrent que l'amélioration du rendement en grain des lignées s'est faite sans augmentation du rendement en biomasse, avec des indices de récolte qui passent de 0,35 à 0,50 environ. Ils citent des résultats similaires obtenus sur cette espèce par d'autres auteurs (dont Van DOBBEN, 1962 : SYME, 1970 ; JaIN \& KulShreSTHA, 1976). De même, dans une comparaison de 8 variétés modernes et anciennes en condition de culture intensive, GRIGNAC et al. (1982) montrent que les rendements en paille et 
en matière sèche totale sont supérieurs chez les variétés anciennes de blé, alors que les rendements en grain sont supérieurs chez les variétés modernes, avec une augmentation des indices de récolte qui passent de 0,34 à 0,45 . Sur fourrages pérennes, ALLERIT (1985) met en évidence, à partir des essais du réseau CTPS, un progrès voisin de $0,05 \mathrm{t} / \mathrm{ha} /$ an pour le trèfle violet, de 0,04 et $0,03 \mathrm{t} / \mathrm{ha} /$ an pour les ray-grass anglais et italien, un progrès nul pour la luzerne et le dactyle et un progrès négatif atteignant $-0,05 \mathrm{t} / \mathrm{ha} / \mathrm{an}$ pour la fétuque élevée, lié dans ce cas à une amélioration de la valeur alimentaire.

Ces résultats confirment aussi que la sélection du maîs destiné à l'ensilage de la plante entière doit faire l'objet de programmes originaux à côté de ceux consacrés à la sélection pour le grain qui ne maîtrisent pas les évolutions possibles d'indice de récolte. Il est d'ailleurs clairement établi que la productivité en plante entière est autant liée au rendement en grain qu'au rendement de la partie non grain, et que la variabilité pour le rendement de la partie non grain est environ le double de celle que l'on observe pour la partie grain (GALLAIS et al., 1981 ; HUNTER, 1985). Ceci rejoint d'ailleurs les travaux de HUNTER (1985) qui montrent que la sélection de familles $S_{1}$ sur des essais de rendement en plante entière au stade ensilage est plus efficace pour améliorer la productivité en plante entière que la sélection des familles $S_{1}$ sur des essais de rendement en grain. Ces résultats montrent également qu'il y a parmi les lignées anciennes une variabilité pour la productivité en plante entière à étudier sur une base plus large, à introduire dans les populations en sélection récur- rente et éventuellement dans des départs en sélection généalogique à base étroite en croisement par exemple avec du matériel classique amélioré pour la résistance à la verse en végétation et l'adaptation aux fortes densités. C'est de génotypes triés sur la productivité en biomasse à partir de populations-source originales que seront issues à moyen terme les familles permettant des progrès notables en sélection pour la valeur fourragère et céréalière, parce que, à partir d'un certain niveau d'indice de récolte, l'augmentation corrélative de biomasse est nécessaire, et parce qu'il semble plus facile de modifier l'indice de récolte que d'augmenter la productivité en plante entière. La sélection du maïs ensilage doit ainsi se situer plutôt en amont de la sélection grain, mais cela n'est pas contradictoire avec l'exploitation de la variabilité génétique issue de la sélection pour la productivité en grain.

Pour ce qui est des progrès à venir d'une manière générale et parmi les voies envisageables, il y a l'augmentation des durées de surface foliaire (DAYNARD \& TOLLENAAR, 1983), l'augmentation de la résistance au stress hydrique, l'augmentation de la résistance aux faibles températures et des capacités de reprises de croissance consécutives, l'augmentation des capacités de photosynthèse aux températures moyennes (STAMP, 1985). L'amélioration de la résistance à la verse en végétation et de la qualité de l'enracinement aura également un effet favorable sur la productivité en biomasse ou en grain.

Reçu le 11 février 1986 Accepté le 18 octobre 1986.

\section{RÉFÉRENCES BIBLIOGRAPHIQUES}

\begin{abstract}
Allerit R., 1985. Espèces fourragères pérennes. Bilan d'observations, progrès liés à la sélection et évolution variétale appréciés à travers l'expérimentation officielle. Mémoire Ingénieur diplômé par l'état, ENSA de Montpellier, $46 \mathrm{p}$.
\end{abstract}

Austin R. B., Bingham J., Blackwell R. D., Evans L. T., Ford M. A., Morgan C. L., Taylor M., 1980. Genetic improvements in winter wheat yields since 1900 and associated physiological changes. $J$. Agric. Sci. Camb., 94, 675-689.

Bachacou J., Masson J. P., Millier C., 1981. Amance 81, I.N.R.A.C.R.F., 516 pages.

Castleberry R. M., Crum C. W., Krull C. F., 1984. Genetic yield improvement of U.S. maize cultivars under varying fertility and climatic environments. Crop. Sci., 24, 33-36.

Daynard T. B., Tollenaar M., 1983. Perspectives d'amélioration des maïs précoces. In : Colloque physiologie du maîs, I.N.R.A. éd., 574 p., 535-567.

Derieux M., Darrigand M., Gallais A., Barrière Y., Bloc D., Montalant J., 1986. Estimation du progrès génétique réalisé chez le maïs grain en France au cours des trente dernières années. $C$. $R$. Acad. Agric. France, 72 (3), 215-222.

Derieux M., Darrigand M., Gallais A., Barrière Y., Bloc D., Montalant Y., 1987. Estimation du progrès génétique réalisé chez le mais grain en France entre 1950 et 1985. Agronomie, 7 (1), 1-11.

Duvick D. N., 1984. Genetic contribution to yield gains of U.S. hybrid maize, 1930 to 1980 . In Genetic contributions to yield gains of five major crop plants W. R. FEHR ed. Crop Science Society of America special publication $\mathrm{n}^{\circ} 7$, Madison Washington.

Gallais A., Vincourt P., Huguet L., 1981. Objectifs et critères de sélection du maïs fourrage. Colloque Eucarpia Maïs-sorgho, Montreux, Suisse.
Grignac P., Poux J., Tomas A., 1982. Comparaison de variétés anciennes et modernes de blé tendre à divers niveaux d'intensification dans un environnement méditerranéen. C. R. Acad. Agric. France, 67, 1434-1453.

Hunter R. B., 1985. Selecting hybrids for silage maize production. A Canadian experience. Proceedings $13^{\mathrm{e}}$ Colloque Eucarpia maîssorgho. 9-12 septembre 1985, Wageningen, Netherlands. P. 140-146.

Jain H. K., Kulshrestha V. P., 1976. Dwarfing genes and breeding for yield in bread wheat. Zeitschrift für Pflanzenzüchtung, 76, 102112.

Lila M., Barrière Y., Traineau R., 1986. Mise au point et étude d'un test enzymatique de la digestibilité de fourrages pauvres ou riches en amidon. Agronomie, 6 (3), 285-291.

Russel W. A., 1984. Agronomic performance of maize cultivars representing different eras of breeding. Maydica, 29, 375-390.

Stamp P., 1985. Chilling stress in maize. Proceedings $13^{e}$ Colloque Eucarpia maiss-sortho, 9-12 septembre 1985 Wageningen, Netherlands. P. 43-60.

Syme J. R., 1970. A high yielding Mexican semi-dwarf wheat and the relationship of yield to harvest index and others varietal characteristics. Australian J. Exp. Agric. Ani. Husb., 10, 350-353.

Tilley J. M. A., Terry R. A., 1963. A two stage technique for the " in vitro " digestion of forage crops. J. Brit. Grassl. Soc., 18, 104111.

Van Dobben W. H., 1962. Influence of temperature and light conditions on dry matter distribution, development rate and yield in arable crops. Netherlands J. Agric. Sci., 10, 377-389. 